\title{
Phase II study of paclitaxel with 3-h infusion in patients with advanced gastric cancer
}

\author{
Kensei Yamaguchi ${ }^{1}$, Masahiro Tada ${ }^{1}$, Noboru Horikoshi ${ }^{2}$, Toru Otani ${ }^{3}$, Hiroya Takiuchi ${ }^{4}$, Soh Saitoh ${ }^{5}$, \\ Ryunosuke Kanamaru ${ }^{6}$, Yasushi Kasai ${ }^{7}$, Wasaburo Koizumi ${ }^{8}$, Yuh Sakata ${ }^{9}$, and Tetsuo Taguchi ${ }^{10}$ \\ and the Paclitaxel Gastric Cancer Study Group in Japan \\ ${ }^{1}$ Department of Gastroenterology, Saitama Cancer Center Hospital, 818 Komuro, Ina-machi, Saitama 362-0806, Japan \\ ${ }^{2}$ Department of Medical Oncology, Cancer Institute Hospital, Japanese Foundation for Cancer Research, Tokyo, Japan \\ ${ }^{3}$ Third Department of Gastroenterology, Osaka Medical Center for Cancer and Cardiovascular Diseases, Osaka, Japan \\ ${ }^{4}$ Second Department of Internal Medicine, Osaka Medical College, Takatsuki, Japan \\ ${ }^{5}$ Department of Gastroenterology, Aomori Prefectural Central Hospital, Aomori, Japan \\ ${ }^{6}$ Department of Clinical Oncology, Tohoku University, Sendai, Japan \\ ${ }^{7}$ Second Department of Surgery, Nagoya University, Nagoya, Japan \\ ${ }^{8}$ Department of Gastroenterology/Medical Oncology, Kitasato University East Hospital, Sagamihara, Japan \\ ${ }^{9}$ Misawa Municipal Hospital, Misawa, Aomori, Japan \\ ${ }^{10}$ Society for Cancer Chemotherapy, Osaka, Japan
}

\begin{abstract}
Background. To increase the options for agents for gastric cancer chemotherapy, we performed a phase II clinical trial on the use of a 3-h infusion of paclitaxel to confirm its efficacy and the feasibility of its use in patients with advanced gastric cancer.

Methods. Thirty-two (32) patients with measurable metastatic gastric cancer were enrolled in this study. Seventeen patients $(53 \%)$ had received prior chemotherapy for metastatic disease, 4 patients $(13 \%)$ had adjuvant chemotherapy alone, and 11 patients $(34 \%)$ were chemotherapy-naive.

Paclitaxel was intravenously infused for $3 \mathrm{~h}$, at a dose of $210 \mathrm{mg} / \mathrm{m}^{2}$, once every 3 weeks. To prevent hypersensitivity reactions, standard premedication was administered to all patients.

Results. Nine (28\%; 9/32) objective partial responses (PRs) were observed $(95 \%$ confidence interval [CI], 14\%-47\%), and the remaining 23 patients showed stable (12 patients; $37.5 \%$ ) and progressive disease (11 patients; $34.4 \%$ ). The median time to response was 20 days (range, 14-38 days). The median response duration was 87 days (range, 50-103 days). The median survival of all patients was 234 days (range, 13-646+ days). The major adverse reactions were myelosuppression (grade 3/4 leukopenia and neutropenia were observed in $59 \%$ and $88 \%$ of the patients, respectively), alopecia, and peripheral neuropathy. Peripheral neuropathy was observed in 19 patients, however, most of the patients recovered after the completion of treatment.

Conclusion. A 3-h infusion of paclitaxel is an effective therapy for advanced gastric cancer and is clinically well tolerated by the patients.
\end{abstract}

Offprint requests to: $\mathrm{K}$. Yamaguchi

Received: October 22, 2001 / Accepted: February 21, 2002
Key words Advanced gastric cancer - Paclitaxel - Phase II study

\section{Introduction}

In digestive tract cancers, anticancer agents have been considered to be less effective in gastric cancer than in cancers in other parts of the tract. Recently, though, some effective agents for gastric cancer have been available; the median survival time is still within the 6-9 months' range, and chemotherapy has been used mainly in the palliative setting for this disease [1].

Response rates of $18 \%-53 \%$ had been obtained in the first-line chemotherapy for gastric cancer. Most chemotherapy regimens for gastric cancer consist of combination chemotherapy that combines drugs with different mechanisms of action, such as: fluorouracil (5-FU)/leucovorin (LV), FAM (5-FU + adriamycin [ADR] + mitomycin C [MMC]), ELF (etoposide [VP16] + LV + 5-FU), FAMTX (5-FU + ADR + methotrexate $[\mathrm{MTX}]), \mathrm{FP}(5-\mathrm{FU}+$ cisplatin [CDDP]), and ECF (epirubicin + CDDP $+5-\mathrm{FU})$. These regimens have played an important role in the progress of chemotherapy for gastric cancer. They have had an impact on efficacy and survival in the chemotherapy of advanced gastric cancer.

Taxol is a novel taxane drug that was originally isolated from Taxus brevifolia, one of the western yews. Unlike the conventional antitumor drugs, it is characterized by the ability to promote the assembly of microtubules [2]. 
Paclitaxel, a newer taxare, has demonstrated anticancer activity against a variety of cancers, including breast cancer [3], ovarian cancer [4], melanoma [5] and lung cancer [6]. In addition, the growth inhibitory effect of paclitaxel on primary cultures of gastric cancer is greater than that of CDDP and ADR [7]. Based on these findings, paclitaxel administration is expected to provide a clinical advantage (or usefulness) in the treatment of gastric cancer.

The recommended optimal dose of paclitaxel in Japan was determined to be $210 \mathrm{mg} / \mathrm{m}^{2}$, and this dose was higher than those in other countries $\left(175 \mathrm{mg} / \mathrm{m}^{2}\right)$. Evaluation of efficacy in patients with gastric cancer yielded a response rate of $20 \%(3 / 15)$ in an early phase II study by Ohtsu et al. [8]. The main adverse drug reaction was myelosuppression (grade 4 neutropenia developed in $67 \%$ of the patients). Based on the above findings, this study was planned to evaluate the efficacy and safety of paclitaxel in the treatment of patients with gastric cancer.

\section{Patients and methods}

\section{Eligibility criteria}

Patients with histologically confirmed gastric cancer were eligible for registration, and the patient inclusion criteria included the following: (1) maximum of one prior chemotherapy regimen for metastatic disease and/ or one adjuvant chemotherapy regimen completed 4 weeks before entry, (2) adequate bone marrow function (white blood cell [WBC] count between $\leq 4000 / \mathrm{ml}$ and $<10000 / \mathrm{ml}$; neutrophil count, $\leq 2000 / \mathrm{ml}$; platelet count, $\geq 100000 / \mathrm{ml}$; hemoglobin, $\leq 9.0 \mathrm{~g} / \mathrm{dl}$ ), adequate liver function (serum bilirubin level, $\leq 1.5 \mathrm{mg} / \mathrm{dl}$ and serum transaminase level, $\leq$ twice the upper limit of the normal range; if hepatic metastasis had been documented, then serum transaminase level, $\leq$ three times the upper limit of the normal range), adequate renal function (serum creatinine level, $\leq 1.5 \mathrm{mg} / \mathrm{dl}$; blood urea nitrogen level, $\leq 25 \mathrm{mg} / \mathrm{dl}$ ), normal electrocardiogram (ECG), (3) Eastern Clinical Oncology Group (ECOG) performance status of 2 or less, (4) age between 20 and 75 years, (5) no other severe medical conditions, (6) no other active malignancy, (7) life expectancy more than 2 months, and (8) detection of a metastatic lesion greater than $1 \mathrm{~cm}$ in diameter.

Written informed consents, in accordance with government guidelines ("Good Clinical Practice" by the Ministry of Health and Welfare of Japan), were also acquired. This study was approved by the Ethics Committees at the participating sites.

\section{Treatment}

To prevent hypersensitivity reactions, all patients were premedicated with $20 \mathrm{mg}$ of dexamethasone given intravenously 12 and $6 \mathrm{~h}$ prior to paclitaxel infusion, and $50 \mathrm{mg}$ of diphenhydramine, given orally, and $50 \mathrm{mg}$ of ranitidine, given intravenously $30 \mathrm{~min}$ prior to paclitaxel infusion. Paclitaxel, $210 \mathrm{mg} / \mathrm{m}^{2}$, was intravenously infused over a 3-h period. Patients received paclitaxel once every 3 weeks unless disease progression or intolerable toxicity was observed. If, in the previous course, the WBC count fell below $1000 / \mathrm{ml}$ or the platelet count decreased to less than $25000 / \mathrm{ml}$, or if a grade 3 or higher nonhematologic toxicity was observed, the paclitaxel dose was reduced to $180 \mathrm{mg} / \mathrm{m}^{2}$. However, if the same reactions were seen with the reduced dose, the dose was further decreased to $150 \mathrm{mg} / \mathrm{m}^{2}$. If the same reactions were still seen even after these dose reductions, the patient was taken off the study.

\section{Evaluation}

The Japan Clinical Oncology Group (JCOG) toxicity criteria (conforming to the National Cancer Center Common Toxicity Criteria [NCC-CTC]) were applied to evaluate adverse drug reactions. Tumor response to chemotherapy was evaluated by the standard World Health Organization (WHO) criteria. The evaluation criteria were as follows: complete response (CR), all lesions disappeared for 4 weeks or more; partial response (PR), the size of all measured lesions was reduced by $50 \%$ or more for 4 weeks or more, in addition to no new lesion appearing and no enhancement of the lesions concerned; stable disease (SD), the size of all lesions was not reduced by $50 \%$ and simultaneously no tumor growth by $25 \%$ or more was seen; and progressive disease (PD), development of a new lesion, not detected previously, or growth of an existing lesion by an estimated $25 \%$ or more. Tumor evaluations were performed by computed tomographic (CT) scans performed during every course.

\section{Statistics}

This study was designed to test the null hypothesis that the true response probability is less than the clinically in significant level of $10 \%$. If it is rejected, we will accept the specified alternative hypothesis that the true response probability is at least a target level of $20 \%$ [9]. Therefore, the sample size was 60 patients, with alpha $=$ 0.05 , beta $=0.2$. This study was designed to be completed earlier than originally planned if reproducibility of the results of a preceding phase II study of paclitaxel for gastric cancer was confirmed [10].

Case registration was completed when the response rate was over the target level of $20 \%$ and the Bayesian 
estimated response probability exceeding the clinically insignificant level of $10 \%$ was over $99 \%$. We produced the latter probability by applying Bayes' theorem [11] using, Jeffreys prior distribution [12]. Survival was calculated from the date of initiation by the Kaplan-Meier method.

\section{Results}

\section{Patient characteristics}

Thirty-two patients were entered in this trial between September 1997 and November 1999; the patient characteristics are summarized in Table 1 . The response rate was more than the target level of the expected response rate at $20 \%$, and the Bayesian estimated response probability exceeding the clinically insignificant level of $10 \%$ became extremely high, at more than $99 \%$, in the 32 registered patients. Thus, the patient registration was completed with the entered 32 patients. All 32 patients were evaluated for response and toxicity in the intentto-treat analysis. The two courses stipulated in the protocol had been completed in 30 of the 32 patients. Of the 2 patients who discontinued the protocol, 1 patient died of disseminated intravascular coagulation (DIC) before entering the second cycle, and another patient had initiated subsequent treatment before confirmation of stable disease (SD).

The median age of the patients was 59 years (range, 32 to 73 years). Sixteen patients $(50 \%)$ had gastric cancer as the primary lesion and 16 patients $(50 \%)$ had undergone surgical resection of primary gastric cancer. Twenty-nine patients (91\%) had an ECOG performance status of 0 or 1 . Histologically, 17 patients $(53 \%)$ had intestinal-type adenocarcinoma, 14 patients (44\%)

Table 1. Patient characteristics

\begin{tabular}{lc}
\hline Characteristic & $n$ \\
\hline Patients & 32 \\
Median age, years (range) & $(32-73)$ \\
Male/Female & $25 / 7$ \\
Performance status & \\
0 & 21 \\
1 & 8 \\
2 & 3 \\
Histologic type & \\
Intestinal & 17 \\
Diffuse & 14 \\
Adenosquamous & 1 \\
Primary surgical resection & 16 \\
Prior chemotherapy & \\
Chemotherapy & 17 \\
Adjuvant chemotherapy only & 4 \\
No chemotherapy & 11 \\
\hline
\end{tabular}

had the diffuse type, and 1 patient (3\%) had adenosquamous carcinoma. All patients had measurable metastatic lesions, and the metastatic site was the lung in 3 patients $(9 \%)$, liver in 21 patients $(66 \%)$, lymph nodes in 21 patients $(66 \%)$, and other areas (bone, adrenal gland, residual stomach, and pelvis) in 4 patients $(13 \%)$. Twenty-one patients $(66 \%)$ received prior chemotherapy that was completed 4 or more weeks prior to entry: 4 received adjuvant chemotherapy alone, and 17 received chemotherapy for metastatic disease. All of the 21 patients who were previously treated with chemotherapy received antimetabolite therapy, and 17 of the 21 patients received platinum-based therapy. Eleven patients were chemotherapy-naive.

\section{Efficacy}

There were nine PRs, 12 SD, nine PD, and two were not evaluable (NE), yielding a response rate of $28 \%$ ( 9 of 32 patients; $95 \%$ confidence interval [CI], 14\%-47\%). The response rates of the patients with metastasis to lung, liver, lymph node, and other sites were $0 \%(0 / 3), 33 \%$ (6/18 evaluable), 16\% (3/19 evaluable), and 25\% (1/4), respectively (Table 2). The response rates of the patients with intestinal-type adenocarcinoma, diffuse-type adenocarcinoma, and adenosquamous carcinoma were $24 \%(4 / 17), 36 \%(5 / 14)$, and $0 \%(0 / 1)$, respectively. The response rate of the 11 patients without prior chemotherapy was $36 \%(4 / 11)$ and that of the 19 evaluable patients with prior chemotherapy was $26 \%(5 / 19)$. The median time to response was 20 days (range, 14 to 38 days) and the median response duration was 87 days (range, 30 to 103 days). The median survival time of all patients was 234 days (95\% CI, 147 to 303 days) (Fig. 1).

\section{Toxicity}

All patients were evaluable for adverse reactions. The total number of treatment courses was 86 (median, 2 courses per patient; range, 1-9 courses). No patient needed dose reduction to $180 \mathrm{mg} / \mathrm{m}^{2}$. The most common adverse drug reactions are summarized in Table 3 . The major adverse reactions were myelosuppression, alopecia, and peripheral neuropathy. Leukopenia and neutropenia were observed in all of the patients, and were grade 3 or more in $59 \%(19 / 32)$ and $88 \%(28 / 32)$ of patients, respectively. Granulocyte colony-stimulating factor (G-CSF) was used in 50\% of the courses (43/86). Mild to moderate myalgia and arthralgia were also observed, in 13 and 17 patients, respectively. Hypersensitivity reactions (skin rash) developed in 5 patients (grade 1 in 3 patients and grade 2 in 2 patients); however, the patients all recovered after appropriate treatment, and the paclitaxel therapy were not discontinued. Although most of the adverse reactions were resolved 
Table 2. Response

\begin{tabular}{|c|c|c|c|c|c|c|c|}
\hline Patients & $(n)$ & CR & PR & SD & PD & $\mathrm{NE}$ & $\begin{array}{l}\text { Response } \\
\text { rate }(\%)\end{array}$ \\
\hline Overall & 32 & 0 & 9 & 12 & 9 & 2 & 28 \\
\hline \multicolumn{8}{|l|}{ Metastatic site ${ }^{a}$} \\
\hline Lung & 3 & 0 & 0 & 1 & 2 & 0 & 0 \\
\hline Liver & 21 & 0 & 6 & 8 & 4 & 3 & 33 \\
\hline Lymph node & 21 & 0 & 3 & 13 & 3 & 2 & 16 \\
\hline Others & 4 & 0 & 1 & 1 & 1 & 0 & 25 \\
\hline \multicolumn{8}{|l|}{ Histologic type } \\
\hline Intestinal & 17 & 0 & 4 & 10 & 2 & 1 & 24 \\
\hline Diffuse & 14 & 0 & 5 & 2 & 6 & 1 & 36 \\
\hline Adenosquamous & 1 & 0 & 0 & 0 & 1 & 0 & 0 \\
\hline \multicolumn{8}{|l|}{ Prior chemotherapy } \\
\hline Adjuvant only & 4 & 0 & 2 & 2 & 0 & 0 & 50 \\
\hline Both adjuvant and metastatic & 3 & 0 & 1 & 2 & 0 & 0 & 33 \\
\hline Metastatic only & 14 & 0 & 2 & 5 & 5 & 2 & 17 \\
\hline No chemotherapy & 11 & 0 & 4 & 3 & 4 & 0 & 36 \\
\hline
\end{tabular}

CR, Complete response; $\mathrm{PR}$, partial response; $\mathrm{SD}$, stable disease; $\mathrm{PD}$, progressive disease; NE, not evaluable

${ }^{a}$ Metastatic sites may not add up to the number of patients, as each patient may have more than one metastatic site

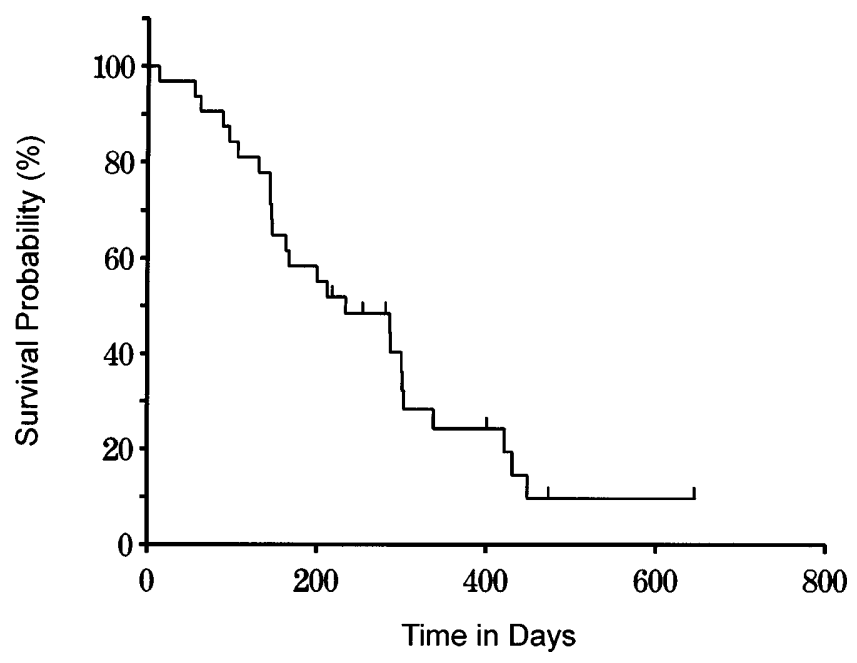

Fig. 1. Overall survival of the 32 patients

after the completion of the study treatment, $42 \%$ of the patients $(8 / 19)$ with peripheral neuropathy continued to experience this adverse reaction after treatment completion. The drug therapy was discontinued in 2 patients due to treatment-related adverse reactions: in 1 patient for peripheral neuropathy, and in the other for liver function disorders. One case of treatment relateddeath, one case of grade 4 liver dysfunction, and one case of grade 4 interstitial pneumonia were reported. The study-drug-related death was due to DIC. In this patient, fever and neutropenia were observed on day 8 (paclitaxel administration day, day 1). The patient received antibiotic therapy and other symptomatic measures, but developed DIC as a complication on day 9 and died on day 12 .

\section{Discussion}

This study examined the safety and efficacy of an infusion of paclitaxel $\left(210 \mathrm{mg} / \mathrm{m}^{2} ; 3-\mathrm{h}\right.$ infusion $)$, given once every 3 weeks, for advanced gastric cancer. This therapy yielded a response rate of $28.1 \%$ ( 9 PRs $/ 32$ patients: $95 \%$ CI, $13.7 \%-46.7 \%$ ); thus, in terms of efficacy, it was placed after S-1 (tegafur/gimestat/otostat potassium; $49 \%)$ [13] and MMC (30\%) [14,15], but ahead of 5-FU (21\%) [16], ADR (13\%-24\%) [14,17], CDDP (19.4\%$22 \%$ ) [18,19], irinotecan hydrochloride (CPT-11; $23.3 \%$ ) [20], and docetaxel (24\%) [21]. These figures represent the results of first-line therapies, excluding those of MMC and CDDP, suggesting that paclitaxel (first-line, 36.4\%; second-line, $23.8 \%$ ) would be extremely useful for the treatment of advanced gastric cancer. In an Eastern Cooperative Oncology Group (ECOG) study, the response rate for paclitaxel $\left(250 \mathrm{mg} / \mathrm{m}^{2}, 24-\mathrm{h}\right.$ infusion) was as low as $4.5 \%$, and it was concluded that this drug was not useful for the treatment of gastric cancer [22]. Using the same method of administration as ours, Ohtsu et al. [8] reported that the response rate was $20 \%(3 / 15)$ among 15 patients, and an other study by Ohtsu et al. [10] reported a response rate of $23.3 \%(14 / 60)$ in 60 patients, including those who received paclitaxel as second-line therapy. Cascinu et al. [23] also used paclitaxel with a similar method $\left(225 \mathrm{mg} / \mathrm{m}^{2}, 3-\mathrm{h}\right.$ infusion $)$, and obtained a response rate of $22.9 \%(8 / 35)$. Thus, the discrepancy 
Table 3. Adverse reactions

\begin{tabular}{|c|c|c|c|c|c|}
\hline \multirow[b]{2}{*}{ Adverse reaction } & \multicolumn{4}{|c|}{ Grade (no. of patients) } & \multirow{2}{*}{$\begin{array}{c}\text { Grades } 3 \text { anc } \\
4(\%)\end{array}$} \\
\hline & 1 & 2 & 3 & 4 & \\
\hline \multicolumn{6}{|l|}{ Hematological } \\
\hline Leucopenia & 1 & 12 & 17 & 2 & 59 \\
\hline Neutropenia & 0 & 4 & 5 & 23 & 88 \\
\hline Anemia & 9 & 12 & 6 & 0 & 19 \\
\hline Thrombocytopenia & 1 & 0 & 2 & 1 & 9 \\
\hline \multicolumn{6}{|l|}{ Non-hematological } \\
\hline Nausea/vomiting & 10 & 6 & 0 & 0 & 0 \\
\hline Diarrhea & 4 & 3 & 0 & 0 & 0 \\
\hline Infection & 4 & 4 & 2 & 1 & 9 \\
\hline Fever & 7 & 7 & 0 & 0 & 0 \\
\hline Hypersensitivity reactions & 3 & 2 & 0 & 0 & 0 \\
\hline Alopecia & 23 & 7 & 0 & 0 & 0 \\
\hline Arrhythmia & 4 & 0 & 0 & 0 & 0 \\
\hline Hypotension & 1 & 1 & 0 & 0 & 0 \\
\hline Peripheral neuropathy & 15 & 4 & 0 & 0 & 0 \\
\hline Myalgia & 9 & 4 & 0 & 0 & 0 \\
\hline Arthralgia & 9 & 8 & 0 & 0 & 0 \\
\hline
\end{tabular}

between the results of the ECOG study and our study may be derived in large part from the difference in the method of administration.

In our study, all patients developed leukopenia or neutropenia (grade 3 or more, $59.4 \%$ and $87.5 \%$, respectively) associated with myelosuppression. Although these effects could be controlled by the administration of G-CSF, they underline the need for caution. Peripheral neuropathy, a side effect specific to paclitaxel, occurred in $19(59.4 \%)$ patients. Although no patient had serious neuropathy, the incidence of this reaction tended to increase with repeated administration of the drug. Improvements were, however, noted in 11 of these 19 patients $(57.9 \%)$ after the end of therapy, with the remaining patients also showing a tendency toward improvement. Although the development of hypersensitivity reactions to polyoxyethylated castor oil (Cremophor EL; BSAF, North Mount Olive, NJ, USA) used as the solvent for this drug, was a concern, there was no case of hypersensitivity reaction to this solvent, as premedication was administered.

The reason for the low median number of dosing courses (2) is considered to be that a large number of patients discontinued the study drug treatment prior to the disease worsening because of a change in treatment policy.

There was one case of treatment-related death (TRD), presumably resulting from infection due to neutropenia; all the other patients were manageable.

The median survival time (MST) in our study was 7.8 months, although the fact that the study included 21 patients who had received previous chemotherapy, and although the study drug was administered as monotherapy. From these results, it can be expected that an effective regimen for gastric cancer will be developed with a combination of paclitaxel and other $\operatorname{drug}(\mathrm{s})$.

In recent years, a weekly regimen of paclitaxel, taking into consideration its mechanism of action, has been widely examined. A report of a comparative study of a weekly infusion vs an infusion once every 3 weeks [24] documented almost the same efficacy, and decreased adverse reactions (in particular, myelosuppression and peripheral neuropathy) with the weekly infusion, although the studies were not conducted in gastric cancer patients. Thus, even though, as mentioned above, there has been no such study in gastric cancer patients, a weekly regimen is of great interest in view of its safety.

In conclusion, the issues to be discussed in regard to paclitaxel are: (1) the establishment of an optimal regimen for monotherapy, and (2) the establishment of combination regimens for use in gastric cancer, based on the optimal regimen for monotherapy. Because paclitaxel has high efficacy even when administered as monotherapy, and because it has been reported to be safe, even for prolonged and repeated administration in breast cancer, it offers great promise as a candidate component of standard regimens for the treatment of gastric cancer.

Acknowledgments We would like to thank the following doctors for their contribution, as committee members of the Paclitaxel Gastric Cancer Study Group in Japan, in evaluating the efficacy and safety of this treatment: Akira Wakui, Tohoku University; Nagahiro Saijyo, National Cancer Center Hospital; Yasuo 
Ohashi, Tokyo University; Tsuneo Sasaki, Tokyo Metropolitan Komagome Hospital; Hiroshi Saito, Yamagata Prefecture Central Hospital; and Kuniaki Shirao, National Cancer Center Hospital. We would also like to thank the following participating doctors: Toshinobu Sato and Junichi Koeda, National Hirosaki Hospital; Masanori Terashima, Iwate Medical University; Takashi Yoshioka, Tohoku University; Haruhiko Sato, Sendai Shakaihoken Hospital; Kouichi Hasegawa and Yoshiki Muto, Tsuboi Hospital; Yasushi Mitachi, Sendai Kosei Hospital; Keisuke Aiba, Yoshinori Ito, Nobuyuki Mizunuma, and Minoru Nakane, Japanese Foundation for Cancer Research; Motonori Sairenji, Kanagawa Cancer Center; Kouichi Hosokawa, Suwa Central Hospital; Osamu Saito, Nagoya University; Noriya Uedo and Hiroyuki Narahara, Osaka Medical Center for Cancer and Cardiovascular Diseases; Kenichi Katsu, Osaka Medical College; Kouichi Yasutake and Takao Tamura, Hyogo Medical Center for Cancer and Cardiovascular Diseases; Tetsuya Toge and Takafumi Kin, Hiroshima University; and Shuji Nakano and Kenji Mitsugi, Kyushu University. This study was supported by a grant from Bristol-Myers Squibb K.K., Tokyo, Japan.

\section{References}

1. Ajani JA, Fairweather J, Dumas P, Patt YZ, Pazdur R, Mansfield PF. Phase II study of taxol in patients with advanced gastric carcinoma. Cancer J Sci Am 1998;4:269-74.

2. Wani MC, Taylor HL, Wall ME, Coggon P, McPhail AT. Plant antitumor agents. VI. The isolation and structure of taxol, a novel antileukemic and antitumor agent from Taxus brevifolia. J Am Chem Soc 1971;93:2325-7.

3. Holmes FA, Walters RS, Theriault RL, Forman AD, Newton LK, Raber MN, et al. Phase II trial of taxol, an active drug in the treatment of metastatic breast cancer. J Natl Cancer Inst 1991;83:1797-805.

4. Einzig AI, Wiernik PH, Sasloff J, Runowicz CD, Goldberg GL. Phase II study and long-term follow-up of patients treated with taxol for advanced ovarian adenocarcinoma. J Clin Oncol 1992; 10:1748-53.

5. Einzig AI, Hochster H, Wiernik PH, Trump DL, Dutcher JP, Garowski E, et al. A phase II study of taxol in patients with malignant melanoma. Invest New Drug 1991;9:59-64.

6. Chang AY, Kim K, Glick J, Anderson T, Karp D, Johnson D. Phase II study of taxol, merbarone, and piroxantrone in stage IV non-small-cell lung cancer: The Eastern Cooperative Oncology Group Results. J Natl Cancer Inst 1993;85:388-94.

7. Matsuoka H, Yano K, Saito T, Seo Y, Tomoda H. Cytotoxicity of paclitaxel in comparison with other anticancer agents against neoplastic cells obtained from clinical gastrointestinal carcinoma tissue. Anticancer Res 1995;15:2001-6.
8. Ohtsu A, Boku N, Tamura F, Muro K, Shimada Y, Saigenji K, et al. An early phase II study of a 3-h infusion of paclitaxel for advanced gastric cancer. Am J Clin Oncol 1998;21:41619

9. Guidelines for the clinical evaluation of antineoplastic drugs. Tokyo: Yakuji Nippo; 1992.

10. Ohtsu A, Shirao K, Miyata Y, Hyodo I, Saito H, Taguchi T. A phase II study of 3-h infusional paclitaxel in patients with advanced gastric cancer. Proc Am Soc Clin Oncol 2000;19:303a (abstract 1194).

11. Savage LJ. The foundation of statistics. New York: Wiley; 1954.

12. Jeffreys H. Theory of probability. 3rd ed. Berkeley: University of California Press; 1961.

13. Sakata Y, Ohtsu A, Horikoshi N, Sugimachi K, Mitachi Y, Taguchi T. Late phase II study of novel pral fluoropyridine anticancer drug S-1 (1 M tegafur- $0.4 \mathrm{M}$ gimestat- $1 \mathrm{M}$ otastat Potassium) in advanced gastric cancer patients. Eur J Cancer 1998;34:1715-20.

14. Gastrointestinal Tumor Study Group. Phase II-III chemotherapy studies in advanced gastric cancer. Cancer Treat Rep 1979; 63:1871-83.

15. Moetrel CG, Lavin PT. Phase II-III chemotherapy studies in advanced gastric cancer. Cancer Treat Rep 1979;63:18639 .

16. Cocconi G, DeLisi V, DiBlasio B. Randomized comparison of 5FU alone or combined with mitomycin and cytarabine (MFC) in the treatment of advanced gastric cancer. Cancer Treat Rep 1982;66:1263-6.

17. Levi JA, Fox RM, Tattersall MH, Woods RL, Thomson D. Analysis of prospectively randomized comparison of doxorubicin versus 5-fluorouracil, doxorubicin, and BCNYU in advanced gastric cancer: implications for future studies. J Clin Oncol 1986;4: $1348-55$.

18. Lacave AJ, Izarzugaza I, Anton-Aparicio LM, Valle Pereda M, Garcia Marco JM, Buesa JM. Phase II clinical trial of cisdichlorodiammineplatinum in gastric cancer. Am J Clin Oncol 1983;6:35-8.

19. Lacave AJ, Wils J, Diaz-Rubio E, Clavel M, Planting A, Bleiberg $\mathrm{H}$, et al. Cisplatinum as second-line chemotherapy in advanced gastric adenocarcinoma: a phase II study of the EORTC gastrointestinal tract cancer cooperative group. Eur J Cancer Oncol 1985;21:1321-4.

20. Futatsuki K, Wakui A, Nakao I, Sakata Y, Kamibe M, Shimada Y, et al. Late phase II study of irinotecan hydrochloride (CPT-11) in advanced gastric cancer. CPT-11 Gastrointestinal Cancer Study Group. Jpn J Cancer Chemother 1994;21:1033-8.

21. Sulkes A, Smyth J, Sessa C, Dirix LY, Vermorken JB, Kaye S, et al. Docetaxel (taxotere) in advanced gastric cancer: results of a phase II clinical trial. Br J Cancer 1994;70:380-3.

22. Einzig AI, Wiernik PH, Lipsitz S, Benson III AB. Phase II trial of taxol in patients with adenocarcinoma of the upper gastrointestinal tract: The Eastern Cooperative Oncology Group results. Proc Am Soc Clin Oncol 1993;12:194 (abstract 566).

23. Cascinu S, Graziano F, Cardarelli N, Marcellini M, Giordani P, Menichetti ET, et al. Phase II study of paclitaxel in pretreated advanced gastric cancer. Anticancer Drugs 1998;9:307-10.

24. Andersson H, Boman K, Ridderheim M, Rosenberg P, Sorbe B, Puistola U, et al. An updated analysis of a randomized study of single agent paclitaxel $(\mathrm{P})$ given weekly vs every 3 weeks to patients (pts) with ovarian cancer $(\mathrm{OV})$ treated with prior platinum therapy. Proc Am Soc Clin Oncol 2000;19:380a (abstract 1505). 